\title{
Fault models for quantum mechanical switching networks
}

\author{
J.D. Biamonte, ${ }^{*}$ J.S. Allen and M.A. Perkowski
}

August 15, 2018

\begin{abstract}
The difference between faults and errors is that, unlike faults, errors can be corrected using control codes. In classical test and verification one develops a test set separating a correct circuit from a circuit containing any considered fault. Classical faults are modelled at the logical level by fault models that act on classical states. The stuck fault model, thought of as a lead connected to a power rail or to a ground, is most typically considered. A classical test set complete for the stuck fault model propagates both binary basis states, 0 and 1, through all nodes in a network and is known to detect many physical faults. A classical test set complete for the stuck fault model allows all circuit nodes to be completely tested and verifies the function of many gates. It is natural to ask if one may adapt any of the known classical methods to test quantum circuits. Of course, classical fault models do not capture all the logical failures found in quantum circuits. The first obstacle faced when using methods from classical test is developing a set of realistic quantum-logical fault models. Developing fault models to abstract the test problem away from the device level motivated our study. Several results are established. First, we describe typical modes of failure present in the physical design of quantum circuits. From this we develop fault models for quantum binary circuits that enable testing at the logical level. The application of these fault models is shown by adapting the classical test set generation technique known as constructing a fault table to generate quantum test sets. A test set developed using this method is shown to detect each of the considered faults.
\end{abstract}

${ }^{*}$ The authors are with Portland State University, 1900 SW Fourth Avenue, P.O. Box 751, Portland, Oregon 97201, USA. JDB present address: Oxford University Computing Laboratory, Wolfson Building, Parks Road, Oxford, OX1 3QD, UK. 


\section{Introduction}

Test methods in use today began to be developed in the 1960's anticipating circuit sizes that would make exhaustive test methods intractable. Classical test theory attempts to determine if a given circuit is or is not functional $[1,2]$. This is accomplished by testing a circuit in order to determine if any of the logical failures modelled by the set of considered fault models are present. Fault models are typically inspired by physical failures but may also represent abstractions that enable the creation of test sets detecting an abundance of actual physical faults.

Applying established methods of classical circuit test theory to test quantum circuits has attracted interest in recent times [3], with preliminary results reported in $[4,5,6,7]$. Despite interest, adequate justification has not been given for any fault model considered. For instance, much current research has assumed that the classical stuck fault model impacts quantum circuits. The classical stuck fault model does not capture non-deterministic and non-localized quantum mechanical faults. It is even possible to develop classical test sets for reversible circuits complete for the stuck fault model, that never turn on a gate [21].

Experimental physicists who build quantum circuits have not experienced much need to research optimized testing methods due to the size of the currently attainable qubit count so the quantum test problem remains of theoretical interest today $[9,10,11,12]$. In appendix $B$ we review both this currently practiced (exhaustive) approach to the quantum circuit test problem as well as distance measures to compare quantum states.

A quantum circuit is built out of gates and these are connected using nodes and wires. If so many as a single gate, single node or a wire is broken, the quantum circuit is unusable. This is in contrast to quantum errors corrected by control codes [45]. The possibility to construct quantum networks completely free of faults is generally assumed since error correction relies on fault free networks. It is therefore important to test the inter-connectivity of the network nodes, wires and gates in an attempt to locate faults. This work addresses this problem by justifying several logical fault models dependent on the structure of quantum switching networks. Test sets complete for logical fault models determine if any considered fault is present in the interconnections or the gates of a quantum network. In practice, test sets are developed to detect all of the most common faults.

Classically, one defines a testability measure as a product of observability and controllability. The controllability of testing a circuit corresponds to propagating a specific input test vector through a network, such that it will map a test vector to a place of fault. This represents an added challenge in the case of testing quantum circuits, since inputs may become entangled and faults may occur non-deterministically. Furthermore, depending on the measurement basis chosen, certain faults may not be detectable. With these limitations in mind and after defining our notation next, in $\S 2$, we present a set of quantum fault models ( 33 through $\S 8$ ). The presence of even one of these faults will make a quantum circuit unusable so we show how to develop test sets in $\S \mathrm{C}$ under the assumption that the fault-tolerant quantum circuit [45] under test contains at most one of the considered faults. We further assume that each quantum circuit is executed multiple times and that the output is averaged over, using a majority voting procedure. ${ }^{1}$

\section{Background and notation}

Let us define the state $\sqrt{2} \cdot| \pm\rangle=|0\rangle \pm|1\rangle$ and mention that the notational conventions from the textbook by Nielsen and Chuang [22] are used. ${ }^{2}$ We let $\hat{Z}$ be the standard observable in the computational basis and $\hat{X}$ be an observable in the conjugate basis $[30] .{ }^{3}$ We present a set of physically motivated quantum fault

\footnotetext{
${ }^{1}$ The Chernoff Bound asserts that polynomial many repetitions of independent samples will converge exponentially fast to the true mean. See for instance chapter 4 of the text book by R. Motwani and P. Raghavan, "Randomized Algorithms," Cambridge University Press, (1995).

${ }^{2}$ Note to readers new to quantum circuits: The background to read this paper appears in Chapters 1,3 and 8 of the text book [22]. Course lecture notes accompanying [22] can be found online [23].

${ }^{3}$ The conjugate basis is formed by measuring $\hat{Z}$ after rotating all qubits with a Hardamard transform [22].
} 
models for quantum circuits built from $k-\mathrm{CN}$ gates [22]. To separate the general from the particular, a focus is made on error and fault models that are independent of implementation. Without loss of generality examples from several current technologies such as liquid state nuclear magnetic resonance spectroscopy $(\mathrm{NMR})^{4}$ and optical quantum computation are used. Although the NMR implementation is not scalable, it is currently the most successful [37]. Optical quantum computation has the potential of scalability and has recently made progress. For instance, in 2003 O'Brien et al. successfully demonstrated an optical quantum CN gate [43]. This gate was improved and further characterized in 2004 by both O'Brien et al. [28] and White et al. [38].

\subsection{Testing Definitions}

In quantum error correcting codes, fault locations are between circuit stages, ${ }^{5}$ and have quantifiable error probabilities [45]. For example, consider the five stage circuit shown in Fig. 1. The numbered locations of possible gate external faults are illustrated by placing an " $\times$ " on the lines representing qubits. The five gates, initial states $\left(\left|\psi_{0}\right\rangle,\left|\psi_{1}\right\rangle,\left|\psi_{2}\right\rangle\right)$ and measurements $\left(M_{0}, M_{1}, M_{2}\right)$ may also contain errors.

Definition 2.1. Error Location: The wire locations between stages as well as any node or gate in a given network represent error locations.

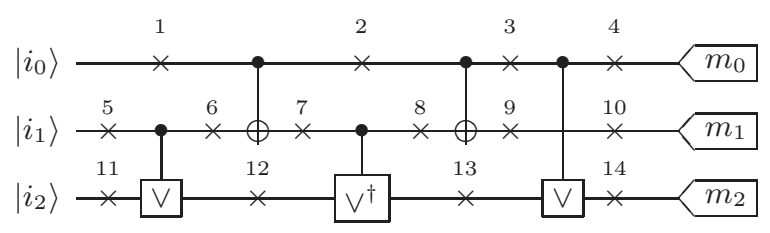

Figure 1: 2-CN gate with 14 gate external error locations numbered above an " $\times$ " and five possibly erroneous gates. The construction of the gates used in this circuit are outlined in Sec. 6 .

Definition 2.2. Gold Circuit: An ideal quantum circuit denoted $G C$. Typically a non-ideal quantum circuit is denoted $Q C$.

Definition 2.3. Fault Set: Denote by $F_{q}$ a set containing all considered faults assumed to impact $Q C$.

Definition 2.4. Quantum Test Set: A sequence of initial states $\left|\psi_{i}\right\rangle$ and corresponding measurements $M_{i}$ used to distinguish $Q C$ possibly perturbed by any $f \in F_{q}$ from a gold circuit $G C$.

Complete fault coverage occurs if executing a test set can determine that all of the considered faults are not present in a given circuit.

Definition 2.5. Fault Coverage: Denote by $Q C$ a quantum circuit possibly perturbed by any element of a set of faults $f \in F_{q}$ and a test set $T$ complete for all $f \in F_{q}$. Fault Coverage occurs for fault $f$ by experimentally running $t \subseteq T$ that detects $f$. A quantum test set that detects all considered faults is a complete test set.

Since even the presence of one of the faults considered in this work would make a quantum circuit unusable, when developing quantum test sets in $\S 9$, we assume that the fault-tolerant quantum circuit [45] under test contains at most one of the considered faults. This assumption is called the quantum single fault model. Classically, test sets complete for single faults are known to detect the presence of multiple faults [70]. Whether or not the quantum single fault model dominates multiple quantum faults is left as an open problem.

\footnotetext{
${ }^{4}$ See Jones [39] for an introduction geared towards those new to NMR Quantum Computing.

${ }^{5}$ In particular see [45] where Knill, Laflamme, and Zurek justified the idea of an error location for the purpose of quantum error correction.
} 
Definition 2.6. Quantum Single Fault Model: Consider the quantum fault set $F_{q}$. In the quantum single fault model, test plans are developed to detect all $f \in F_{q}$ assuming that at most one fault, $f$, is present in $Q C$.

Definition 2.7. Pauli Matrices:

$$
\begin{gathered}
\sigma_{x}=|1\rangle\langle 0|+| 0\rangle\left\langle 1\left|, \quad \sigma_{y}=i\right| 0\right\rangle\langle 1|-i| 1\rangle\langle 0|, \\
\sigma_{z}=|0\rangle\langle 0|-| 1\rangle\langle 1| \quad \text { and } \quad \sigma_{i}=|0\rangle\langle 0|+| 1\rangle\langle 1| .
\end{gathered}
$$

Definition 2.8. Rotation Matrices:

$$
\begin{gathered}
R_{x}(\theta)=\left(\begin{array}{cc}
\cos (\theta / 2) & -i \cdot \sin (\theta / 2) \\
-i \cdot \sin (\theta / 2) & \cos (\theta / 2)
\end{array}\right), \quad R_{y}(\theta)=\left(\begin{array}{cc}
\cos (\theta / 2) & -\sin (\theta / 2) \\
\sin (\theta / 2) & \cos (\theta / 2)
\end{array}\right) \\
\text { and } \\
R_{z}(\phi)=e^{-i \phi / 2}|0\rangle\left\langle 0\left|+e^{i \phi / 2}\right| 1\right\rangle\langle 1| .
\end{gathered}
$$

With the basic definitions behind us, the coming sections discuss gate level failures and introduce several requirements that a quantum test set must satisfy. Each requirement must be satisfied for a test set to be complete and cover all considered faults.

\section{$3 \quad$ Faults modelled with Pauli matrices}

Definition 3.1. Pauli Fault Model: The addition of an unwanted Pauli matrix $f$ in quantum network $Q C$, at error location $l$ and with placement probability $p$.

The unavoidable entanglement between a quantum processor and the outside world is described by many authors as, "coupling to an initially independent environment [49]." This is a primary source of decoherence. A large amount of research has been devoted to removing the local effects of decoherence by quantum error correcting codes. Consequently, what are known as error models are found in the quantum error correcting code literature $[44,50,51,52,53]$. The most investigated error model is the "independent depolarizing error [45]." This model has the effect of completely randomizing a given qubit with some probability [22] and "...error models designed to control depolarizing errors apply to all independent error models [49]." These codes are designed to correct unwanted single qubit $\sigma_{x}, \sigma_{y}$ and $\sigma_{z}$ rotations. The following is a list of the range of errors modeled assuming Pauli Faults, with some supporting references:

- Depolarizing Channels [22, 49]

- Amplitude Dampening [22, 45]

- Phase Damping $[22,54]$

- Phase-Flips $[22,45,54]$

- Bit-Flips $[22,45]$

- Initialization Inaccuracies [54, 55]

- Measurement Inaccuracies $[31,56,57]$

In addition to noise, repeatable errors in a physical construction lead to another class of faults addressed in the literature. These are known as systematic errors and are again modeled by Pauli Faults. Systematic errors are closer to the types of errors that classical test engineers refer to as faults. These errors are described by Cummins and Jones as, "arising from the reproducible imperfections in the apparatus used to implement quantum computations [58]." The most common systematic errors are given in the following list, again with supporting references:

- Pulse Length Errors [35, 58, 61, 62]

- Off-Resonance Effects [58, 35, 47] 
- Refocusing Errors $[63,47]$

Quantum Fault Model 1. (Pauli Fault Model 1) A bit flip $\left(\sigma_{x}\right.$ or $\left.\sigma_{y}\right)$ at any error location must be detectable.

It turns out that these faults are very easy to detect in quantum switching circuits. The following Theorem (3.2) states that any $\sigma_{x}$ or $\sigma_{y}$ fault occurring in a network built from $k-\mathrm{CN}$ gates is detectable with any computational basis input state. In addition, a reversible system preserves information and one can show that the probability of detection for fault $f$ observable with $\hat{A}$, is directly related to the probability of $f$ 's presence.

Theorem 3.2. Pauli Faults $\sigma_{x}$ and $\sigma_{y}$ impacting an $n$ qubit network $Q C$ comprised of $k-C N$ gates at any gate external error location are detected with any basis input state $|s\rangle$ given an observable in the computational basis.

Proof. Any reversible binary quantum network $Q C$ of $l$ qubits and $n$ stages bijectively maps each input $|s\rangle$ to a unique output $\left|s^{\prime}\right\rangle$. Each gate $g \in Q C$ is reversible, thus any stage $n$ acting on input vector $|s\rangle$ corresponds to exactly one output vector at the $(n+1)^{t h}$ stage. A qubit flip $\left(f_{p}\right)$ occurs before or after any stage $n$ on any wire $l$, changing the output from the previous stage (and input to the next). Any $\sigma_{x}$ or $\sigma_{y}$ fault is therefore detectable based on the properties of reversibility [64] with any basis state input $|k\rangle$ given an observable in the computational basis.

From Theorem 3.2 it follows that any test set that contains a basis state input and corresponding measurement in the computational basis dominates a test set complete for Pauli Fault Model 1. This desirable property does not hold for $\sigma_{z}$ phase faults.

Quantum Fault Model 2. (Pauli Fault Model 2) A phase flip $\left(\sigma_{z}\right)$ at any error location must be detectable.

\section{Initialization Faults}

Initialization faults were discussed in detail by Kak [54], and addressed experimentally in [55, 47]. Because initialization accuracies relate to a machine's ability to perform a task (such as not altering the initial state population in NMR [47]), one can develop a test set to determine if the machine is impacted by any of the considered initialization faults. We model these faults using Def. 4.1.

Definition 4.1. Initialization Error: A qubit with an initial state impacted by an unwanted rotation $R_{n}(\theta)$ where $n \in\{x, y, z\}$, or a qubit that is only correctly prepared in one basis state and not the other.

From Def. 4.1, examples of how initialization faults spread are shown in Fig. 2. The fault in Fig. 2 (b) could occur when the desired initial state is $|01 c\rangle$ and the top qubit is inverted $(|c\rangle \rightarrow|\bar{c}\rangle)$ resulting in the state: $\cos \theta|01 c\rangle-i \sin \theta|11 \bar{c}\rangle$. After being acted on by the $2-\mathrm{CN}$ gate, the state of the system becomes $\cos \theta|01 c\rangle-i \sin \theta|11 c\rangle$. There is now a probability of $(\sin \theta)^{2}$ that an incorrect value will be measured.

A similar scenario holds for Fig. 2 (c) - in this case the center qubit is impacted by an initialization fault as opposed to the top qubit in Fig. 2 (b). In Fig. 2 (d) the desired initial state is $|--+\rangle$, however a fault impacts the bottom qubit flipping its phase changing the initial state to $|---\rangle$. Now the $2-\mathrm{CN}$ gate will entangle the state of the system incorrectly, resulting in state $(|00\rangle-|01\rangle-|10\rangle-|11\rangle)|-\rangle$. This fault can be detected with a test set detecting unwanted instances of the Pauli Faults. A second type of initialization error will now be discussed.

Generally, there exists a certain set of states left invariant under a quantum operation [22]. For example, qubit preparation may be altered with a form of amplitude dampening. Thus, a faulty qubit might only 

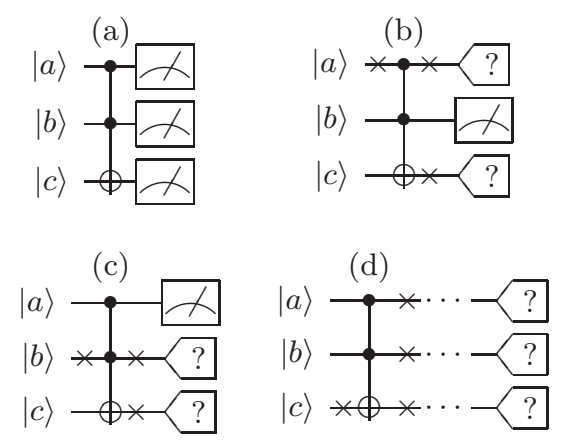

Figure 2: Initialization Errors Impacting a 2-CN Gate: (a) correct circuit, (b)-(d) various initialization errors.

allow preparation into one state. A qubit that can only be prepared in density state $|0\rangle\langle 0|$ is modeled with the following operation elements:

$$
\begin{gathered}
E_{0}=|0\rangle\langle 0|+(\sqrt{1-\gamma})| 1\rangle\langle 1| \\
E_{1}=|0\rangle\langle 0|+(\sqrt{\gamma})| 1\rangle\langle 1|
\end{gathered}
$$

Consider a register prepared in density state $\rho=\rho_{0} \otimes \ldots \otimes \rho_{k} \otimes \ldots \otimes \rho_{n}$. The $k^{t h}$ qubit is desired to start the computation in an arbitrary state, expressed as:

$$
\rho=\rho_{0} \otimes \ldots \otimes\left(\begin{array}{cc}
\alpha^{2} & \alpha \beta^{*} \\
\beta \alpha^{*} & \beta^{2}
\end{array}\right) \otimes \ldots \otimes \rho_{n}
$$

The unwanted impact of initial state dampening is expressed as: $\mathcal{E}(\rho)=\sum_{k} \sigma_{i} \otimes \ldots \otimes E_{k} \otimes \ldots \otimes \sigma_{i} \cdot \rho \cdot \sigma_{i} \otimes$ $\ldots \otimes E_{k}^{\dagger} \otimes \ldots \otimes \sigma_{i}$. This results in the state:

$$
\rho^{\prime}=\rho_{0} \otimes \ldots \otimes\left(\begin{array}{cc}
\alpha^{2}+\gamma \beta^{2} & \alpha \beta^{*} \sqrt{1-\gamma} \\
\beta \alpha^{2} \sqrt{1-\gamma} & \beta^{2}(1-\gamma)
\end{array}\right) \otimes \ldots \otimes \rho_{n} .
$$

The projection of the $k^{\text {th }}$ qubit's state onto the basis $|1\rangle\langle 1|$ is forced into basis state $|0\rangle\langle 0|$ and the off diagonal terms are suppressed (both based on some parameter $\gamma$ ). Similarly, a faulty qubit might only allow preparation into density state $|1\rangle\langle 1|$. A test set complete for Quantum Fault Model 3 determines if any qubit can only be correctly prepared in one basis state and not the other.

Quantum Fault Model 3. (Initialization Fault Model) Each qubit must be initialized in both basis states $|0\rangle$ and $|1\rangle$.

\section{Lost Phase Faults}

Shenvi, Brown and Whaley [53] studied Grover's search algorithm [22] impacted by random phase errors in the oracle. ${ }^{6}$ They model errors by applying unwanted phase shifts $\pm \epsilon$ to the state of a quantum register marked by an oracle: $\mathcal{O}:|k\rangle \longrightarrow e^{i \pi \cdot(f(k) \pm \epsilon)}|k\rangle$. Several references including [53] call this a "phase-kickerror." Fig. 3 (b), (c) and (d) illustrate faulty controls that have phase kick-back faults. A correct 3-CN gate will map an input state $|+++\rangle|-\rangle$ to output state $(|000\rangle+|001\rangle+|010\rangle+|011\rangle+|100\rangle+|101\rangle+$ $|110\rangle-|111\rangle)|-\rangle$. Each term in the superposition that activates the gate will undergo a phase shift of $|n\rangle \longrightarrow e^{i \pi}|n\rangle$. If the fault in Fig. 3 (b) is present, the circuit's output is $(|000\rangle+|001\rangle+|010\rangle-|011\rangle+$

\footnotetext{
${ }^{6}$ Grover's original algorithm has recently been updated by L. Grover [65]. Although this new "Fixed Point Algorithm" is more robust, it is still subject to phase errors. The algorithm has been experimentally verified by Xiao and Jones [66], systematic errors in the physical implementation were briefly explored. B. Reichardt and L. Grover have also recently developed methods of systematic error correction for this new algorithm [67].
} 


\begin{tabular}{c||c|c|c|c|c} 
term & initial & GC (a) & (b) & (c) & (d) \\
\hline$|000\rangle$ & +1 & +1 & +1 & +1 & +1 \\
$|001\rangle$ & -1 & -1 & -1 & -1 & -1 \\
$|010\rangle$ & +1 & +1 & -1 & +1 & +1 \\
$|011\rangle$ & -1 & -1 & +1 & -1 & -1 \\
$|100\rangle$ & +1 & +1 & +1 & -1 & +1 \\
$|101\rangle$ & -1 & -1 & -1 & +1 & -1 \\
$|110\rangle$ & +1 & -1 & -1 & -1 & +1 \\
$|111\rangle$ & -1 & +1 & +1 & +1 & -1
\end{tabular}

Table 1: The impact Phase Faults from Fig 3 have on input state $|++-\rangle$. The first column shows the phase of each term before being acted on by the circuit. The column GC (a) shows the correct phase relative phase of each term in the superposition. The remaining columns (b-d) show how the phase changes depending on the fault present.

$|100\rangle+|101\rangle+|110\rangle-|111\rangle)|-\rangle$. In this case, relative phase shifts occur on both states $|011\rangle$ and $|111\rangle$ since those activate the gate when the top control is broken. Another type of fault is phase damping. This is a noise process altering relative phases between quantum states [22]. A test set complete for the Lost Phase Fault Model determines if the faults described above are present in a given quantum circuit.

Quantum Fault Model 4. (Lost Phase Fault Model) Consider the circuit input as $H^{\otimes n} \cdot\left|x_{1}, x_{2}, \ldots, x_{n}\right\rangle$ with $x_{i} \in\{0,1\}$ and measurement of $\hat{X}$. Given freedom in the choice of input vector $x_{1}, x_{2}, \ldots, x_{n}$ a circuit must be shown to have no missing single control or single gate.

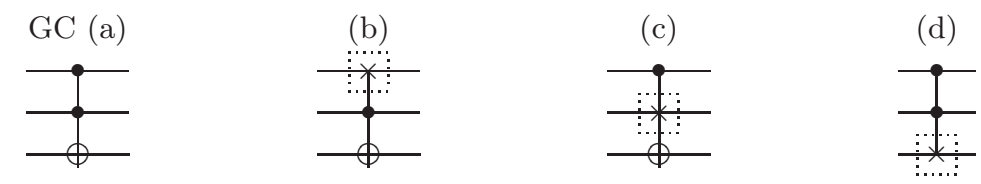

Figure 3: 2-CN Gate and Phase Faults: (a) Gold Circuit, (b) weak top control, (c) weak second control, (d) weak gate.

To show that the considered phase faults are not present in the network we place Hardamard gates at the start and at the end of the circuit under test and measure $\hat{Z}$. Let us denote the circuit under test as $C_{i}$ where subscript $i$ signifies that impact of the $i^{\text {th }}$ considered fault. We will define the input as $\rho=\left|x_{1} x_{2} x_{3}\right\rangle\left\langle x_{1} x_{2} x_{3}\right|$ with $x_{1}, x_{2}$ and $x_{3}$ in $\{0,1\}$. The goal is to show separation of GC from each of the considered faults where $\rho^{\prime}=H^{\otimes 3} \cdot C_{i} \cdot H^{\otimes 3} \cdot \rho \cdot H^{\otimes 3} \cdot C_{i}^{\dagger} \cdot H^{\otimes 3}$ allows freedom in the choice of the input $\rho$. Here we choose $\rho=|001\rangle\langle 001|$ and note that for fault $(\mathrm{b}): \operatorname{tr}\left(|100\rangle\langle 100| \cdot \rho^{\prime}\right)=1$, for fault $(\mathrm{c}): \operatorname{tr}\left(|101\rangle\langle 101| \cdot \rho^{\prime}\right)=1$ and for fault $(\mathrm{d}): \operatorname{tr}\left(|001\rangle\langle 001| \cdot \rho^{\prime}\right)=1$. For GC $\operatorname{tr}\left(|001\rangle\langle 001| \cdot \rho^{\prime}\right)=\operatorname{tr}\left(|100\rangle\langle 100| \cdot \rho^{\prime}\right)=\operatorname{tr}\left(|101\rangle\langle 101| \cdot \rho^{\prime}\right)=$ $\operatorname{tr}\left(|111\rangle\langle 111| \cdot \rho^{\prime}\right)=1 / 4$. To test the considered $2-\mathrm{CN}$ gate for the considered phase faults one must show separation in the above quantities.

\section{Faded Control Faults}

The building blocks needed to implement any quantum algorithm with NMR can be based on single spin rotations and $\mathrm{CN}$ gates [47]. CN gates are realized using a scheme illustrated in Fig. 4. The center gate is a $\mathrm{CZ}$ gate and is built using a $\phi$ gate with angle $\pi$ (see [68], § 3.1 Eqn. 34):

$$
C Z_{i}=|00\rangle\langle 00|+| 01\rangle\langle 01|+| 10\rangle\left\langle 10\left|+e^{i \pi}\right| 11\right\rangle\langle 11| .
$$




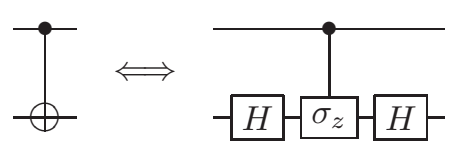

Figure 4: $\mathrm{CN}$ gate constructed with elementary building blocks [68]. The $\mathrm{H}$ gate $\left(H=i R_{y}(\pi / 2) R_{z}(\pi)\right)$ is given as $H=\frac{1}{\sqrt{2}}(|0\rangle+|1\rangle)\left\langle 0\left|+\frac{1}{\sqrt{2}}(|0\rangle-|1\rangle)\langle 1|\right.\right.$ and the center controlled phase shift gate $\left(\mathrm{CZ}_{i}\right)$ is given in Eqn. 3.

In any physical implementation, the $C Z_{i}$ gate might deviate according to our ability to apply phase $e^{i \phi}$ correctly to term $|11\rangle\langle 11|$. This can be represented as:

$$
C Z_{r}=|00\rangle\langle 00|+| 01\rangle\langle 01|+| 10\rangle\left\langle 10\left|+e^{i \phi}\right| 11\right\rangle\langle 11|
$$

An ideal CN gate creates the following mapping: $C N_{i}:|10\rangle \longrightarrow|11\rangle$. If the CZ gate applies a phase at the wrong angle $\phi$, the mapping becomes: $C N_{r}:|10\rangle \longrightarrow\left(1+e^{i \phi}\right)|10\rangle+\left(1-e^{i \phi}\right)|11\rangle$. The fidelity ${ }^{7}$ between the real and ideal $\mathrm{CN}$ gate with input $|10\rangle$ is:

$$
F\left(C N_{i}|10\rangle\left\langle 10\left|C N_{i}^{\dagger}, C N_{r}\right| 10\right\rangle\langle 10| C N_{r}^{\dagger}\right)=\frac{1}{2}(1-\cos \phi) .
$$

Another gate constructed using a $\phi$ gate with angle $\pi / 2$ [68] is known as the CV gate. The $\mathrm{V}$ gate is given as:

$$
\vee=\left|\vee_{0}\right\rangle\left\langle 0|+| \vee_{1}\right\rangle\langle 1|
$$

where $\left|\vee_{0}\right\rangle=(1+i)|0\rangle+(1-i)|1\rangle$ and $\left|\vee_{1}\right\rangle=(1-i)|0\rangle+(1+i)|1\rangle$. The CN and CV gates may be combined to create $2-\mathrm{CN}$ gates as shown in Fig. 5. It turns out that by adjusting $\phi, n^{\text {th }}$ root of NOT gates can be constructed [27]. These can be used to build any $k-\mathrm{CN}$ gate (for instance, by setting $\phi= \pm \pi / 4$ the $4^{\text {th }}$ root of NOT gates can be created and used to build the $3-\mathrm{CN}$ gates in this paper).

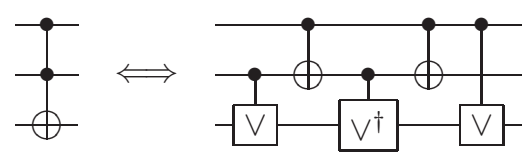

Figure 5: 2-CN gate constructed with elementary building blocks.

Test sets complete for the faded control fault model introduced below must turn each gate on by concurrently activating all controls [21]. These test sets also determine if each control can be turned off properly. A test set complete for the Faded Control Fault Model tests a controls' function with the target in a basis state. It also tests the controls' impact on both activating and non-activating states.

Quantum Fault Model 5. (Faded Control Fault Model) For the target acting on basis state $|0\rangle$ or $|1\rangle$ : All controls must be activated concurrently and each control must be addressed with a non-activating state.

\section{$7 \quad$ Forced Gate Faults}

Forced gate faults are non-unitary. An example of a non-unitary operation is a gate that when activated applies an "amplitude dampening process [22]" to the target bit (a type of relaxation process [47]). A test set complete for Quantum Fault Model 6 forces the gate to act on both a $|0\rangle$ and a $|1\rangle$ to uniquely show that this considered fault is not present. This can be seen further by examining the Truth Table in Fig. 7 . The Forced Gate Fault model is given in Fig. 6.

Quantum Fault Model 6. (Forced Gate Fault Model) Each target must separately act on basis state inputs $|0\rangle$ and $|1\rangle$.

\footnotetext{
${ }^{7}$ See Appendix B for an explanation of the fidelity.
} 


\begin{tabular}{c||c|c|c} 
input & GC (a) & (b) & (c) \\
\hline$|000\rangle$ & $|000\rangle$ & $|000\rangle$ & $|000\rangle$ \\
$|001\rangle$ & $|001\rangle$ & $|001\rangle$ & $|001\rangle$ \\
$|010\rangle$ & $|010\rangle$ & $|0 \underline{1} 1\rangle$ & $|011\rangle$ \\
$|011\rangle$ & $|011\rangle$ & $|01 \underline{0}\rangle$ & $|010\rangle$ \\
$|100\rangle$ & $|100\rangle$ & $|100\rangle$ & $|10 \underline{1}\rangle$ \\
$|101\rangle$ & $|101\rangle$ & $|101\rangle$ & $|10 \underline{0}\rangle$ \\
$|110\rangle$ & $|111\rangle$ & $|111\rangle$ & $|111\rangle$ \\
$|111\rangle$ & $|110\rangle$ & $|110\rangle$ & $|110\rangle$
\end{tabular}

Table 2: Fault table for 2-CN gate with (b) missing top control and (c) missing bottom control. A test set complete for this fault table is as follows: To separate GC from (b) and (c) measure $\hat{Z}$ for the following inputs $|01 \star\rangle$ and $|10 \star\rangle$, with $\star\{0,1\}$.

(a)

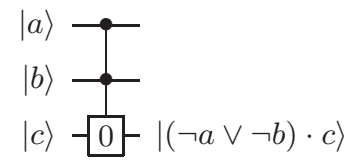

(b)

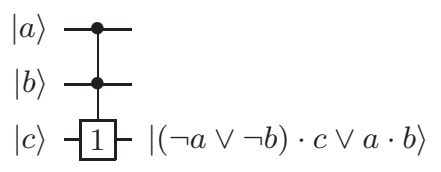

Figure 6: Forced gate Faults: (a) 2-CN gate that correctly acts on a $|1\rangle$ by changing the state to $|0\rangle$, (b) $2-\mathrm{CN}$ gate that correctly acts on a $|0\rangle$ by changing the state to $|1\rangle$. (In both of these cases, we consider binary inputs.)

\section{Measurement Faults}

Certain types of measurement faults can be caused from a "limitation in the sensitivity of a measurement apparatus [56]." Measurement faults are often modelled by the Pauli Fault Model. For example, to project (measure) the state of a photon one places a slit in front of a photo detector. Polarization states inline with the slit will be allowed to reach the photo detector and the angle of the slit is subject to error [31]. Aside from the Pauli Fault Model already considered, a faulty measurement instrument is modelled as a probe that couples to a qubit and consistently returns an a certain value. In Fig. 9 the single measurement fault model is illustrated by placing a faulty measurement gate at the output of the circuit. The truth table derived from Fig. 9 is shown in Fig. 10. The corresponding fault table if given in Fig. 11. Test sets complete for the Measurement Fault Model 7 detect the the faults defined below in Def. 8.1.

Definition 8.1. Measurement Fault Model: A working measurement gate is replaced with a faulty measurement gate that returns only a logic-zero or a logic-one.

Quantum Fault Model 7. (Measurement Fault Model) Each qubit must be measured in both logic-zero and logic-one states.

\section{Conclusion}

In this work we have considered the adaptation of know classical methods to test quantum circuits. The first step in this program is to develop a set of realistic quantum fault models. The fault models we have presented enable circuit testing at the logical, as oposed to currently practiced approach of testing quantum circuits at the device level. Finding additional fault models, that are physically inspired and not dominated by the those introduced here, is an open problem. In the appendix we have included an example test set for a specfic circuit using the fault models presented in this work. 


\begin{tabular}{|c||c|c|c|}
\hline input & GC & $\mathrm{a}$ & $\mathrm{b}$ \\
\hline$|000\rangle$ & 000 & 000 & 000 \\
\hline$|001\rangle$ & 001 & 001 & 001 \\
\hline$|010\rangle$ & 010 & 010 & 010 \\
\hline$|011\rangle$ & 011 & 011 & 011 \\
\hline$|100\rangle$ & 100 & 100 & 100 \\
\hline$|101\rangle$ & 101 & 101 & 101 \\
\hline$|110\rangle$ & 111 & 110 & 111 \\
\hline$|111\rangle$ & 110 & 110 & 111 \\
\hline
\end{tabular}

Figure 7: The Truth Table for 2-CN Gate and the impact of Forced Gate Faults: The column denoted 'input' shows the respective input combinations possible. Columns denoted 'a' and 'b' show the circuit's response given the presence of faults from Fig. 6 (a) and (b), respectively.

\begin{tabular}{|c||c|c|}
\hline input & $\mathrm{a}$ & $\mathrm{b}$ \\
\hline$|000\rangle$ & 0 & 0 \\
\hline$|001\rangle$ & 0 & 0 \\
\hline$|010\rangle$ & 0 & 0 \\
\hline$|011\rangle$ & 0 & 0 \\
\hline$|100\rangle$ & 0 & 0 \\
\hline$|101\rangle$ & 0 & 0 \\
\hline$|110\rangle$ & 1 & 0 \\
\hline$|111\rangle$ & 0 & 1 \\
\hline
\end{tabular}

Figure 8: Fault Table for 2-CN Gate perturbed by the Forced Gate Faults given in Fig. 6. Each binary entry in the fault table corresponds to a single test (row) and a single fault (column). Tests are labeled $|000\rangle$ to $|111\rangle$ and faults are labeled 'a' and 'b', as shown in Fig. 6 (a) and (b). A '1' in the table corresponds to a given test (row) detecting a given fault (column).

\section{Acknowledgments}

The Quantum Circuit diagrams were drawn in LATEX using Q-circuit [73]. The simulation tool QuIDDPro [72] was used during this study. We would like to thank G.F. Viamontes, D. Maslov, J.A. Jones and P.J. Love.

\section{A The Partial Trace}

In this Appendix we review the partial trace [22]. Consider Hilbert space $A$ of system $A \otimes B$. We will trace over system $A$, leaving system $B$ in a mixed state. We have, $\operatorname{tr}_{A}\left(\left|a_{1}\right\rangle\left\langle a_{2}|\otimes| b_{1}\right\rangle\left\langle b_{2}\right|\right)$. Now consider $\left|e_{i}\right\rangle$ as an orthonormal basis for system $A$, we may write the partial trace as, $\sum_{i}\left\langle e_{i}|| a_{1}\right\rangle\left\langle a_{2}|\otimes| b_{1}\right\rangle\left\langle b_{2}|| e_{i}\right\rangle$. After we recall the general fact about tensor products, $|a\rangle\langle b|\otimes| c\rangle\langle d|=| a\rangle|c\rangle\langle b|\langle d|$, it is easy to see a well known equation for the trace of a component part of a composite system, $\sum_{i}\left\langle e_{i}|| a_{1}\right\rangle\left\langle a_{2}|| e_{i}\right\rangle \otimes\left|b_{1}\right\rangle\left\langle b_{2}\right|$ $=\operatorname{tr}\left(\left|a_{1}\right\rangle\left\langle a_{2}\right|\right)\left|b_{1}\right\rangle\left\langle b_{2}\right|$.

\section{B Current Methods Used to Test Quantum Circuits}

In this Appendix we review the main approach currently used to test quantum circuits. In the mid to late 90's experimentalists developed a method of black box characterization known as quantum process tomography [14]. A quantum process is described as a map between input and output quantum states, e.g. $\rho_{\text {out }}=\mathcal{E}\left(\rho_{\text {in }}\right)=\sum_{j} E_{j} \rho_{i n} E_{j}^{\dagger}$, where the map $\mathcal{E}$ is a quantum operation 8 and the operators $E_{j}$ are called

\footnotetext{
${ }^{8}$ In this work we only consider the case where $\sum_{j} E_{j} E_{j}^{\dagger}=I$.
} 
(a)

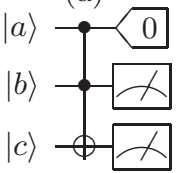

(d)

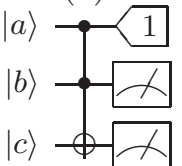

(b)

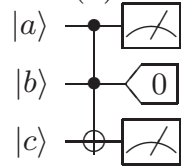

(e)

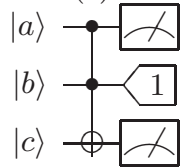

(c)

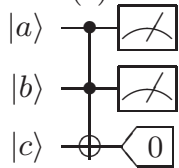

(f)

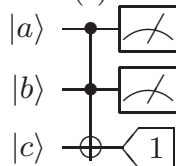

Figure 9: Measurement Errors: Figs (a), (b) and (c) illustrate measurement faults that statistically favor logic-zero. Figs (d), (e) and (f) contain measurement faults statistically favoring logic-one.

\begin{tabular}{|c||c|c|c|c|c|c|c|}
\hline input & GC & a & b & c & d & e & f \\
\hline$|000\rangle$ & 000 & 000 & 000 & 000 & 100 & 010 & 001 \\
\hline$|001\rangle$ & 001 & 001 & 001 & 000 & 101 & 011 & 001 \\
\hline$|010\rangle$ & 010 & 010 & 000 & 010 & 110 & 010 & 011 \\
\hline$|011\rangle$ & 011 & 011 & 001 & 010 & 111 & 011 & 011 \\
\hline$|100\rangle$ & 100 & 000 & 100 & 100 & 100 & 110 & 101 \\
\hline$|101\rangle$ & 101 & 001 & 101 & 100 & 101 & 111 & 101 \\
\hline$|110\rangle$ & 111 & 010 & 100 & 110 & 110 & 110 & 111 \\
\hline$|111\rangle$ & 110 & 011 & 101 & 110 & 111 & 111 & 111 \\
\hline
\end{tabular}

Figure 10: Truth Table for 2-CN Gate Impacted by Measurement Faults. The column denoted 'input' shows the input combinations possible on the amplitude plane. Columns denoted 'a' through 'f' show the circuit's response given the presence of faults from Fig. 9 (a) through (f).

operation elements. ${ }^{9}$ Process tomography is a procedure used to reconstruct the behavior of a quantum network by performing state tomography on a set of initial states $\rho_{i}$ that form an operator basis for the system in question [13]. ${ }^{10}$ The input states and measurement projectors in process tomography each form a basis for the set of $n$-qubit density matrices requiring $d^{2}=2^{2 n}$ elements in each set [22], where $d$ is the dimension of the Hilbert space. For a two-qubit gate $d^{2}=16$, resulting in 256 different settings of input states and measurement projectors. One of many possible input combinations (adapted from the optics experiment in [28]) forming an operator basis needed to characterize the space of two-qubit circuits is the following: ${ }^{11}$

$$
\begin{array}{r}
\left\{|00\rangle,|01\rangle,|10\rangle,|11\rangle,|0+\rangle,\left|0 y_{-}\right\rangle,\left|1 y_{-}\right\rangle,|1+\rangle\right. \\
|++\rangle,\left|y_{+} y_{-}\right\rangle,\left|y_{+}+\right\rangle,\left|+y_{+}\right\rangle,|+1\rangle \\
\left.\left|y_{+} 1\right\rangle,|+0\rangle,\left|y_{+} 0\right\rangle\right\} .
\end{array}
$$

Of course there exist many possible choices for such a basis. In general however, for a system of $n$ qubits the computational basis states $|0\rangle, \ldots,\left|2^{n-1}\right\rangle$ and superpositions $(|q\rangle \pm|r\rangle) / \sqrt{2}$ are prepared, where $q \neq r[32]$.

Given many copies of an experimental sample, state tomography is a procedure allowing one to reconstruct

\footnotetext{
${ }^{9} \mathrm{~A}$ review of the properties of operation elements is given in Ch. 3 of the $1998 \mathrm{PhD}$ Thesis by Nielsen [29].

${ }^{10} \mathrm{~A}$ purely mathematical discussion of process tomography is presented, all measurements are treated as yielding exact probabilities and all sources of error in those measurements are ignored. For experimental background see for example [28] and [30, 31]. Chapter 8 in Ref. [22] also has an introduction to both State and Process Tomography.

${ }^{11}$ Using the notation that: $\left(\left|y_{+}\right\rangle=|0\rangle+i|1\rangle\right.$ and $\left.\left|y_{-}\right\rangle=|0\rangle-i|1\rangle\right)$. The measurement projectors corresponding to this set of initial states adapted from the optics experiment given in [28] are: $\langle 00|,\langle 10|,\langle+1|,\left\langle y_{-} 0\right|,\left\langle y_{-} 1\right|,\langle 11|,\langle 01|,\langle 0-|,\left\langle 0 y_{-}\right|$, $\left\langle y_{-} y_{-}\right|,\left\langle y_{-}-\right|,\langle+-|,\left\langle+y_{+}\right|,\langle 1-|,\left\langle 1 y_{-}\right|$and $\langle+0|$.
} 


\begin{tabular}{|c||c|c|c|c|c|c|}
\hline input & a & b & c & d & e & f \\
\hline$|000\rangle$ & 0 & 0 & 0 & 1 & 1 & 1 \\
\hline$|001\rangle$ & 0 & 0 & 1 & 1 & 1 & 0 \\
\hline$|010\rangle$ & 0 & 1 & 0 & 1 & 0 & 1 \\
\hline$|011\rangle$ & 0 & 1 & 1 & 1 & 0 & 0 \\
\hline$|100\rangle$ & 1 & 0 & 0 & 0 & 1 & 1 \\
\hline$|101\rangle$ & 1 & 0 & 1 & 0 & 1 & 0 \\
\hline$|110\rangle$ & 1 & 1 & 0 & 0 & 0 & 1 \\
\hline$|111\rangle$ & 1 & 1 & 1 & 0 & 0 & 0 \\
\hline
\end{tabular}

Figure 11: Fault table for 2-CN gate derived from Fig. 9. Each binary entry in the fault table corresponds to a single test (row) and a single fault (column). Tests are labeled $|000\rangle$ to $|111\rangle$ and faults are labeled 'a' through 'f'. A value of ' 1 ' in the table corresponds to a given test (row) detecting (covering) a given fault (column).

an arbitrary quantum state to a given accuracy. It requires a set of simple measurement operators that are products of Pauli matrices. The method relies on creating a set of orthogonal measurements and using the Hilbert-Schmidt inner product [22] to expand the state of $\rho$ based on the average outcome of each measurement. A single qubit may be reconstructed as the following density matrix:

$$
\rho=\frac{\operatorname{tr}(\rho) \sigma_{i}+\operatorname{tr}\left(\sigma_{x} \rho\right) \sigma_{x}+\operatorname{tr}\left(\sigma_{y} \rho\right) \sigma_{y}+\operatorname{tr}\left(\sigma_{z} \rho\right) \sigma_{z}}{2} .
$$

Expressions like $\operatorname{tr}\left(\sigma_{x} \rho\right)$ in Eqn. 7 refer to an average measurement outcome where $\sigma_{x}$ is an observable. A similar expansion to that of Eqn. 7 applies to $n$-qubit systems. For example, reconstruction of any two-qubit operator requires a total of $2^{2 n}=16$ measurement observables:

$$
\begin{array}{r}
\left\{\sigma_{i} \otimes \sigma_{i}, \sigma_{i} \otimes \sigma_{x}, \sigma_{i} \otimes \sigma_{y}, \sigma_{i} \otimes \sigma_{z}, \sigma_{x} \otimes \sigma_{i}, \sigma_{x} \otimes \sigma_{x}, \sigma_{x} \otimes \sigma_{y}, \sigma_{x} \otimes \sigma_{z}, \sigma_{y} \otimes \sigma_{i}, \sigma_{y} \otimes \sigma_{x}\right. \\
\left.\sigma_{y} \otimes \sigma_{y}, \sigma_{y} \otimes \sigma_{z}, \sigma_{z} \otimes \sigma_{i}, \sigma_{z} \otimes \sigma_{x}, \sigma_{z} \otimes \sigma_{y}, \sigma_{z} \otimes \sigma_{z}\right\} .
\end{array}
$$

A difficulty associated with quantum process tomography is that in experimental practice, the observables are not easily realized. A system with $d$ dimensions requires $16^{d}-4^{d}$ independent parameters to uniquely describe the process [13], where $d=2^{n}$. The useful method of quantum process tomography was developed out of a need for black box characterization (for that purpose its use appears unavoidable). However, process tomography works independently of the set of gates realized in the network and their possible faults and when used as a method to test quantum switching networks, it has a classical counterpart known as brute-force or complete functional-testing.

Distance measures between quantum states are now reviewed. First we recall the well known Fidelity measure between quantum states.

Definition B.1. The Fidelity between density matrices $\rho$ and $\sigma$ is defined as:

$$
F(\rho, \sigma) \equiv \operatorname{tr}(\sqrt{\sqrt{\rho} \sigma \sqrt{\rho}})^{2}
$$

When $\rho=|\psi\rangle\langle\psi|$ is a pure state the fidelity has an easy interpretation as the overlap between $\rho$ and $\sigma$, reducing to:

$$
F(\psi, \sigma)=\langle\psi|\sigma| \psi\rangle \text {. }
$$

Furthermore, the Fidelity evaluates to zero when two pure states being compared are orthogonal, it evaluates to one when two states being compared are identical, and is not a metric. ${ }^{12}$ For a discussion regarding an operational interpretation of the Fidelity for a mixed state see Reference [33].

\footnotetext{
${ }^{12}$ Two common ways of turning the Fidelity into a metric are the Bures metric, $B(\rho, \sigma) \equiv \sqrt{2-2 \sqrt{F(\rho, \sigma)}}$ and the angle, $A(\rho, \sigma) \equiv \arccos (\sqrt{F(\rho, \sigma)})$, a very comprehensive discussion of these details can be found elsewhere, e.g. Ref. [34].
} 
A second common distance measure is the Trace Distance between quantum states.

Definition B.2. The Trace Distance between density matrices $\rho$ and $\sigma$ is defined as:

$$
D(\rho, \sigma) \equiv \frac{1}{2} \operatorname{tr}|\rho-\sigma|
$$

where $|Z|=\sqrt{Z^{\dagger} Z}$. Since $0 \leq D \leq 1$ the trace distance is a genuine metric on quantum states [34, 22] and thus has the following three properties: $(i) D(\rho, \sigma) \geq 0$ with $D(\rho, \sigma)=0$ iff $\sigma=\rho,($ ii $)$ Symmetry: $D(\rho, \sigma)=$ $D(\sigma, \rho)$, and $($ iii $)$ the Triangle Inequality: $D(\mathcal{E}(\rho), \mathcal{G}(\rho)) \leq D(\mathcal{E}(\rho), \mathcal{F}(\rho))+D(\mathcal{F}(\rho), \mathcal{G}(\rho))$. The Trace Distance represents the statistical distribution between quantum states with respect to measurement. The Trace Distance has the property of contractivity, $D(\mathcal{E}(\rho), \mathcal{E}(\sigma)) \leq D(\rho, \sigma)$ whenever $\mathcal{E}$ is a trace-preserving quantum operation. This just means that acting on arbitrary quantum states $\rho$ and $\sigma$ both with operation $\mathcal{E}$ will never increase how well one can distinguish these states with respect to measurements [34, 22].

The Trace Distance and Fidelity are complementary measures and should be considered equally important when comparing two quantum states [34]. Distance measures may also be used to compare and contrast a real process $\mathcal{F}$ and an ideal process $\mathcal{E}$, such that $\Delta(\mathcal{F}, \mathcal{E})$ defines an error metric on a quantum process [34].

Definition B.3. The S-Fidelity between real quantum process $\mathcal{F}$ and ideal quantum process $\mathcal{E}$ is defined as:

$$
\Delta_{\min }^{F}(\mathcal{F}, \mathcal{E}) \equiv \min _{|\psi\rangle} \Delta(\mathcal{F}(\psi), \mathcal{E}(\psi))
$$

where the minimum is over all possible pure state inputs and $\Delta$ is a Fidelity measure on quantum states.

Definition B.4. The S-Distance between real quantum process $\mathcal{F}$ and ideal quantum process $\mathcal{E}$ is defined as:

$$
\Delta_{\max }^{D}(\mathcal{F}, \mathcal{E}) \equiv \max _{|\psi\rangle} \Delta(\mathcal{F}(\psi), \mathcal{E}(\psi))
$$

where the maximum is over all possible pure state inputs and $\Delta$ is a Distance metric on quantum states.

Instead of considering all pure states, it is helpful to restrict our thinking to a set of inputs needed to form a complete operator basis for the system in question. In this case, experimentally determining the S-Distance/S-Fidelity amounts to performing state tomography on this complete operator basis input set while keeping track of the worst Trace Distance (10)/Fidelity (9) between the reconstructed state and that of the ideal. Ref. [34] stated that, "...the S-Distance and S-Fidelity are the two best error measures, and should be used as the basis for comparison of real quantum information processing experiments to the theoretical ideal."

\section{Testing Example}

In this section we will present the following quantum circuit. Figure 12 shows diagramatically the quantum circuit perturbed by some of the fault models we have introduced here. The interested reader can consult Table $\mathrm{C}$ to consider the test set that will determine if any of the fault models considered in this work are present or not.

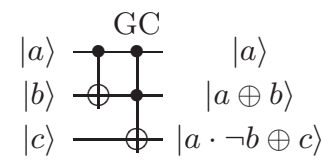




\begin{tabular}{c|c|c|c|c|c|c|c|c}
\hline abc & $G C$ & $f_{1}$ & $f_{2}$ & $f_{3}$ & $f_{4}$ & $f_{5}$ & $f_{6}$ & $f_{7}$ \\
000 & 000 & 010 & 000 & 000 & 000 & 000 & 000 & 000 \\
001 & 001 & 011 & 001 & 001 & 001 & 001 & 001 & 001 \\
010 & 010 & 000 & 011 & 010 & 010 & 010 & 010 & 010 \\
011 & 011 & 001 & 010 & 011 & 011 & 011 & 011 & 011 \\
100 & 111 & 111 & 111 & 101 & 100 & 111 & 110 & 110 \\
101 & 110 & 110 & 110 & 100 & 101 & 110 & 111 & 111 \\
110 & 100 & 100 & 100 & 111 & 100 & 111 & 100 & 101 \\
111 & 101 & 101 & 101 & 110 & 101 & 110 & 100 & 101 \\
\hline
\end{tabular}
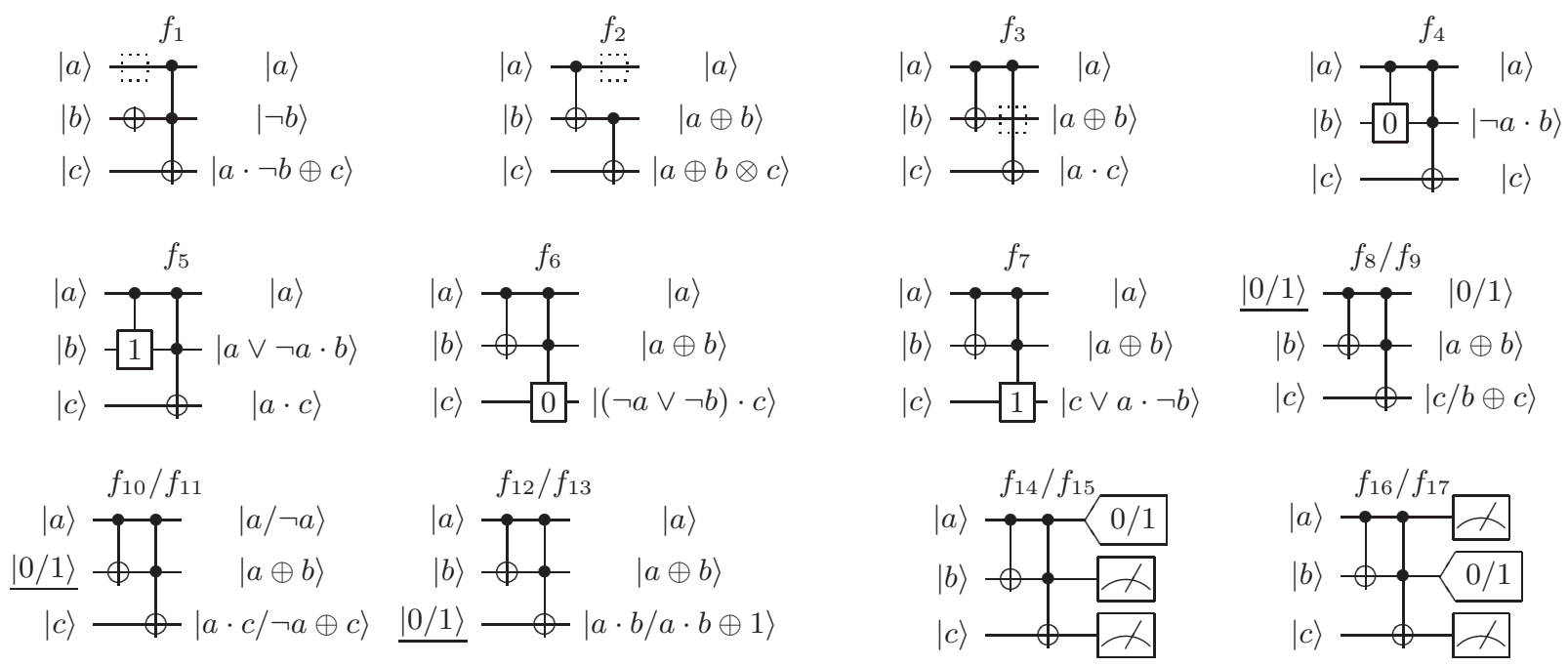
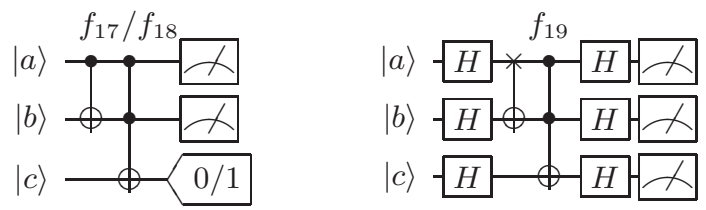
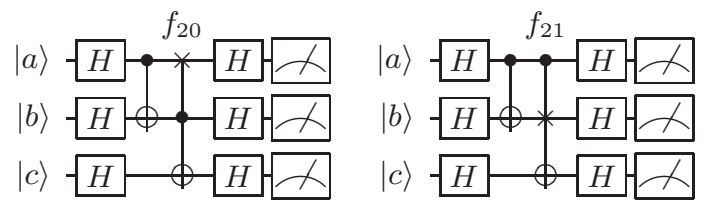

Figure 12: Measurement Errors: Figs (a), (b) and (c) illustrate measurement faults that statistically favor logic-zero. Figs (d), (e) and (f) contain measurement faults statistically favoring logic-one.

\section{References}

[1] W. Kautz, "Automatic fault detection in combinatoinal switching networks," Proc. AIEE $2^{\text {nd }}$ Switching Circuit Theory and Logical Design Symp., 1961, pp. 195-214.

[2] W.H. Kautz, Testing faults in combinational cellular logic arrays, Proceedings of 8th annu. Symp. Switching and Automata Theory, 1971, pp. 161-174.

[3] S.K. Shukla, R. Kam, S.C. Goldstein, F. Brewer, K. Banejee and S. Basu, "Nano, Quantum, and Molecular Computing: Are we Ready for the Validation and Test Challenges?," IEEE Pannel Disscussion, 0-7803-8236-6, 2003, pp. 3-7, online: http://www.ece.ucsb.edu/.

[4] J.D. Biamonte and M.A. Perkowski, "Testing a Quantum Computer," Proceedings of, KIAS-KAIST $5^{\text {th }}$ Workshop on Quantum Information Science, Seoul Korea, August $29^{t h}-31^{\text {st }}, 2004$, pp. 16.

[5] J.P. Hayes, I. Polian and B. Becker, "Testing for Missing-Gate Faults in Reversible Circuits," Proc. Asian Test Symposium, Taiwan, 2004, pp. 100-105. 
[6] M.A. Perkowski et al., "Test Generation and Fault Localization for Quantum Circuits," Proc $35^{\text {th }}$ ISMVL, May 2005, pp. 62-68, DOI : 10.1109/ISMVL.2005.46.

[7] K.N. Patel, J.P. Hayes and I.L. Markov, "Fault Testing for Reversible Circuits," IEEE Trans. on CAD, 23(8), 2004, pp. 1220-1230, quant-ph/0404003.

[8] D. Deutsch, "Quantum computational networks," Proceedings of the Royal Society of London. Series A, Mathematical and Physical Sciences, Volume 425, Issue 1868, 1989, pp. 73-90.

[9] M.H.S. Amin, M. Grajcar, E. II'ichev', A.M. Maassen van den Bringk, G. Rose, A.Y. Smirnov and A.M. Zagoskin. "Superconducting Quantum Storage and Processing," IEEE Interational Solid-State Circuits Conference, ISSCC, Session 16, 2004, 10 pages.

[10] J. Jones, M. Mosca and R. Hansen, "Implementation of a Quantum Search Algorithm on a Nuclear Magnetic Resonance Quantum Computer," Nature Vol. 393, 1998, pp. 344-346, quant-ph/9805069.

[11] J. Jones and M. Mosca, "Approximate quantum counting on an NMR ensemble quantum computer," Phys.Rev.Lett. 83:1050, 1999, 4 pages, quant-ph/9808056.

[12] A.M. Steane and D.M. Lucas, "Quantum computing with trapped ions, atoms and light," Fortschritte der Physik, special issue, 2000, 17 pages, quant-ph/0004053.

[13] A.M. Childs, I.L. Chuang and D.W. Leung, "Realization of quantum process tomography in NMR," Phys. Rev. A Iss. 64, 012314, 2001, 8 pages, quant-ph/0012032.

[14] I.L. Chuang and M.A. Nielsen, "Prescrition for experimental determination of the dynamics of a quantum black box," J. Mod. Opt. 44, 2455, 1997, 6 pages, quant-ph/9610001.

[15] G.M. D'Ariano and P. Lo Presti, "Measuring Experimentally the Matrix Elements of an Arbitrary Quantum Operation," Phys. Rev. Lett. Vol. 86, 2001, 41954198, DOI: 10.1103/PhysRevLett.86.4195.

[16] K. Kim, M. Song, S. Lee, and J-S. Lee, "Quantum Process Tomography with Arbitary Number of Ancillary Qubits in Nuclear Magnetic Resonance," Journal of Korean Physical Soceity (JKPS), 2005.

[17] J.B. Altepeter, D. Branning, E. Jeffrey, T.C. Wei, P.G. Kwiat, R.T. Thew, J.L. O'Brien, M.A. Nielsen and A.G. White, "Ancilla-assisted quantum process tomography," Phys. Rev. Lett. 90, 2003, 4 pages, quant-ph/0303038.

[18] P.W. Shor, "Polynomial-Time Algorithms for Prime Factorization and Discrete Logarithms on a Quantum Computer," Proceedings of the 35th Annual Symposium on Foundations of Computer Science, Santa Fe, NM, Nov. 20-22, 1994, pp 124-134 quant-ph/9508027.

[19] U. Kalay, M.A. Perkowski and D.V. Hall "A Minimal Universal Test Set for Self-Test of EXOR-Sumof-Product Circuits," IEEE Transactions on Computers, vol. 49 no. 3, 2000, pp. 267-276.

[20] L.K. Grover, "Quantum mechanics helps in searching for a needle in a haystack," Physical Review Letters Iss. 2, 79:325, 1997, pp. 325-328 quant-ph/9706033.

[21] J.S. Allen, J.D. Biamonte and M.A. Perkowski, "ATPG for Reversible Circuits using Technology-Related Fault Models," Proc. $7^{\text {th }}$ International Symposium on Representations and Methodology of Future Computing Technologies, RM2005, Tokyo, Japan, September $5^{\text {th }}$ and $6^{\text {th }}, 2005,8$ pages.

[22] M.A. Nielsen and I.L. Chuang, "Quantum Computation and Quantum Information," Cambridge Univ. Press, 2000

[23] M. Oskin, "Quantum Computing Lecture Notes," Class Notes, University of Washington, 2004, cs.washington.edu. 
[24] D. Deutsch, "Video Lectures on Quantum Computation,", 2004, free online, www.quiprocone.org.

[25] A.W. Harrow and M.A. Nielsen, "How robust is a quantum gate in the presence of noise?," Phys. Rev. A Vol. 68, 012308, 2003, 14 pages, quant-ph/0301108.

[26] M. Mosca, "Quantum Computer Algorithms," $\mathrm{PhD}$ thesis, University of Oxford, 1999, http://www.cacr.math. uwaterloo.ca/.

[27] A. Barenco, C.H. Bennett, R. Cleve, D.P. DiVincenzo, N. Margolus, P.W. Shor. T. Sleator, J. Smolin, and H. Weinfurter, "Elementary gates of quantum computation," Phys.Rev.A, Vol. 52(5):3457-3467, 1995, 31 pages, quant-ph/9503016.

[28] J.L. O'Brien, G.J. Pryde, A. Gilchrist, D.F.V. James, N.K. Langford, T. C. Ralph and A. G. White, "Quantum process tomography of a controlled-NOT gate," Phys. Rev. Lett. Vol. 93, 080502, 2004, 4 pages, quant-ph/0402166.

[29] M.A. Nielsen, "Quantum information theory," PhD thesis, University of New Mexico, Report UNM-9808 1998, 259 pages, quant-ph/0011036.

[30] D.W. Leung, "Towards Robust Quantum Computation," PhD thesis, Stanford University, 2000, 243 pages, cs/0012017.

[31] D.F.V. James, P.G. Kwiat, W.J. Munro and A.G. White, "On the Measurement of Qubits," Phys. Rev. A Vol. 64, 052312, 2001, 23 pages, quant-ph/0103121.

[32] M.A. Nielsen, "A simple formula for the average gate fidelity of a quantum dynamical operation," Phys. Lett. A Vol. 3034 , 2002, pp. 249-252, quant-ph/0205035.

[33] J.L. Dodd and M.A. Nielsen, "A simple operational interpretation of the fidelity," Phys. Rev. A Vol. 66, 044301, 2002, 1 page, quant-ph/0111053.

[34] A. Gilchrist, N.K. Langford and M.A. Nielsen, "Distance measures to compare real and ideal quantum processes," Phys. Rev. A Vol. 71, 062310, 2005, 14 pages, quant-ph/0408063.

[35] M.D. Bowdrey and J.A. Jones, "A Simple and Convenient Measure of NMR Rotor Fidelity," JAJ-QP01-01, 2001, 2 pages quant-ph/0103060.

[36] M.D. Bowdrey, D.K.L. Oi, A.J. Short, K. Banaszek and J.A. Jones, "Fidelity of Single Qubit Maps," Phys. Lett. A Vol. 294, 258, 2002, 5 pages, (Supersedes quant-ph/0103090 and quant-ph/0103060), (2002), quant-ph/0201106.

[37] M. Steffen, L.M.K Vandersypen and I.L. Chuang. "Toward Quantum Computation: A Five-Qubit Quantum Processor," IEEE Micro, 2001, pp. 24-34, DOI: 10.1109/40.918000.

[38] A.G. White, A. Gilchrist, G.J. Pryde, J.L. O'Brien, M.J. Bremner and N.K. Langford, ”Measuring Controlled-NOT and two-qubit gate operation," , 2003, 10 pages, quant-ph/0308115.

[39] J.A. Jones, "NMR Quantum Computing," in Quantum Computation and Quantum Information Theory, World Scientific, Singapore 2001, http://nmr.physics.ox.ac.uk;

[40] "Nuclear Magnetic Resonance Quantum Computation," in Quantum Entanglement and Information Processing, D. Esteve, J.-M. Raimond, and J. Dalibard (Eds.), Elsevier Science, 2004, http://nmr.physics.ox.ac.uk.

[41] J.A. Jones and M. Mosca, "Implementation of a Quantum Algorithm to Solve Deutsch's Problem on a Nuclear Magnetic Resonance Quantum Computer," Journal of Chemical Physics Vol. 109, August $1^{\text {st }}$ 1998, pp. 1648-1653, quant-ph/9801027. 
[42] L.M.K. Vandersypen, M. Steffen, G. Breyta, C.S. Yannoni, M.H. Sherwood and I.L. Chuang, "Experimental realization of Shor's quantum factoring algorithm using nuclear magnetic resonance,", Nature Vol. 414, 2001, pp. 883-887, quant-ph/0112176.

[43] J.L. O'Brien, G.J. Pryde, A.G. White, T.C. Ralph and D. Branning, "Demonstration of an all-optical quantum controlled-NOT gate,", Nature Vol. 426, 2003, 5 pages, quant-ph/0403062.

[44] P.W. Shor, "Fault-Tolerant Quantum Computation," $37^{\text {th }}$ Symposium on Foundations of Computing, IEEE Computer Society Press Vol. 37, 1996, pp. 56-65, quant-ph/9605011.

[45] E. Knill, R. Laflamme and W.H. Zurek, "Resilient Quantum Computation: Error Models and Thresholds,", Proc. Mathematical, Physical Engineering Sciences Vol. 454, 1997, pp. 365-384 quant-ph/9702058.

[46] C.H. Bennett, D.P. DiVincenzo, J.A. Smolin, W.K. Wootters, "Mixed State Entanglement and Quantum Error Correction," Phys.Rev. A Vol. 54, 1996, pp. 3824-3851, quant-ph/9604024.

[47] L.M.K. Vandersypen, C.S. Yannoni, and I.L. Chuang, "Liquid State NMR Quantum Computing," Encyclopedia of Nuclear Magnetic Resonance Vol. 9: Advances in NMR, 2002, pp. 687697, http://qt.tn.tudelft.nl.

[48] P.J. Love and B. Boghosian, "From Dirac to Diffusion: Decoherence in Quantum Lattice Gases," To appear in QIP, 2005, quant-ph/0507022.

[49] E. Knill, R. Laflamme, A. Ashikhmin, H. Barnum, L. Viola and W.H. Zurek. "Introduction to Quantum Error Correction,” LA Science Vol. 27, 2002, 22 pages, quant-ph/0207170.

[50] D. Aharonov amd M. Ben-Or, "Fault-Tolerant Quantum Computation With Constant Error Rate," Proc. $29^{t h}$ Ann. ACM Symp. on Theory of Computing, New York, 1998, p. 176, quant-ph/9611025; quant-ph/9906129.

[51] S. Bettelli, "Quantitative model for the effective decoherence of a quantum computer with imperfect unitary operations," Phys. Rev. A, Vol. 69, 042310, 2004, 14 pages, quant-ph/0310152.

[52] A. Barenco, T.A. Brun, R. Schack, and T.P. Spiller, "Effects of noise on quantum error correction algorithms," Mod. Phys. Lett. A, Vol. 13, 1998, pp. 2503-2512, quant-ph/9612047.

[53] N. Shenvi, K.R. Brown and K.B. Whaley, "Effects of Random Noisy Oracle on Search Algorithm Complexity,” Phys. Rev. A, Vol. 68, 052313, 2003, 11 pages quant-ph/0304138.

[54] S. Kak, "The Initialization Problem in Quantum Computing," Foundations of Physics, vol 29, 1999, pp. 267-279, quant-ph/9805002.

[55] M.S. Anwar, D. Bazina, H. Carteret, S.B. Duckett, T.K. Halstead, J.A. Jones, C.M. Kozak and R.J.K. Taylor, "Preparing High Purity Initial States for Nuclear Magnetic Resonance Quantum Computing," Phys. Rev. Lett. Vol. 93, 040501, 2004, 3 pages, quant-ph/0312014.

[56] A.M. Childs, J. Preskill and J. Renes, "Quantum information and precision measurement," J.Mod.Opt. Vol. 47, pp. 155-176, 2000, quant-ph/9904021.

[57] C.P. Williams and S.H. Clearwater, "Explorations in quantum computing," Springer Verlag, 1998.

[58] H.K. Cummins and J.A. Jones, "Use of composite rotations to correct systematic errors in NMR quantum computation," New Journal of Physics, Vol 2 6.1-6.12, 2000, 11 pages, quant-ph/9911072.

[59] S. Lee, J.S. Lee, T. Kim, J.D. Biamonte and M.A. Perkowski, "The Cost of Quantum Gate Primitives," Journal of Multiple-Valued Logic and Soft Computing, 2005. 
[60] G.J. Ni, and S.Q. Chen, "Advanced Quantum Mechanics," Rinton Press Inc, 2002, 465 pp..

[61] H.K. Cummins, G. Llewellyn, and J.A. Jones, "Tackling Systematic Errors in Quantum Logic Gates with Composite Rotation," Phys. Rev. A Vol. 67, 042308, 2003, 7 pages, quant-ph/0208092.

[62] K.M. Obenland and A.M. Despain, "Impact of Errors on a Quantum Computer Architecture," Technical Report, Information Sciences Institute, University of Southern California, Oct 1 ${ }^{\text {st }}$, 1996, http://www.isi.edu/.

[63] J.A. Jones and E. Knill, "Efficient Refocussing of One Spin and Two Spin Interactions for NMR Quantum Computation," J.Magn.Resonance Vol. 141, pp. 322-325, 1999, quant-ph/9905008.

[64] W.H. Zurek, "Reversibility and Stability of Information Processing Systems," Phys. Rev. Lett. Vol. 53, 1984, pp. 391-394, DOI: 10.1103/PhysRevLett.53.391.

[65] L.K. Grover, "A different kind of quantum search," 13 pages, 2005, quant-ph/0503205.

[66] L. Xiao and J.A. Jones, "Error tolerance in an NMR Implementation of Grover's Fixed-Point Quantum Search Algorithm," in press Phys.Rev.A, 2005, 5 pages, quant-ph/0504054.

[67] B.W. Reichardt and L.K. Grover, "Quantum error correction of systematic errors using a quantum search framework," free online, 2005, 6 pages, quant-ph/0506242.

[68] J.A. Jones, R.H. Hansen and M. Mosca, "Quantum Logic Gates and Nuclear Magnetic Resonance Pulse Sequences," J.Magn.Resonance Vol. 135, 1998, pp. 353-360, quant-ph/9805070

[69] P.P. Rohde, G.J. Pryde, J.L. O'Brien and T.C. Ralph, "Quantum gate characterization in an extended Hilbert space," free online, 2004, 4 pages, quant-ph/0411144.

[70] E. J. McCluskey and C.W. Tseng, "Stuck-fault tests vs. actual defects," in Proc. 2000 Int. Test Conf., Atlantic City, 2000, pp. 336343.

[71] D. Maslov, C. Young, D. M. Miller and G. W. Dueck, "Quantum Circuit Simplification Using Templates," In Proc. DATE Conference, Munich, Germany, March 2005, pp. 1208-1213.

[72] G. F. Viamontes, I. L. Markov, J. P. Hayes, "Graph-based simulation of quantum computation in the density matrix representation," Quantum Information and Computation, Vol. 5, No. 2, 2005, pp. 113-130.

[73] B. Eastin and S.T. Flammia, "Q-circuit Tutorial," free online, 2004, 7 pages, quant-ph/0406003. 